ARTICLE

https://doi.org/10.1038/s41467-019-13946-0

\title{
Conversion of a conventional superconductor into a topological superconductor by topological proximity effect
}

\author{
C.X. Trang ${ }^{1}$, N. Shimamura ${ }^{1}$, K. Nakayama (i) ${ }^{1,2}$, S. Souma (10 ${ }^{3,4}$, K. Sugawara (1) ${ }^{1,3,4}$, I. Watanabe ${ }^{1}$, K. Yamauchi ${ }^{5}$, \\ T. Oguchi (1) ${ }^{5}$, K. Segawa ${ }^{6}$, T. Takahashi ${ }^{1,3,4}$, Yoichi Ando ${ }^{7}{ }^{7}$ \& T. Sato ${ }^{1,3,4 \star}$
}

Realization of topological superconductors (TSCs) hosting Majorana fermions is a central challenge in condensed-matter physics. One approach is to use the superconducting proximity effect (SPE) in heterostructures, where a topological insulator contacted with a superconductor hosts an effective $p$-wave pairing by the penetration of Cooper pairs across the interface. However, this approach suffers a difficulty in accessing the topological interface buried deep beneath the surface. Here, we propose an alternative approach to realize topological superconductivity without SPE. In a $\mathrm{Pb}$ (111) thin film grown on $\mathrm{TIBiSe}_{2}$, we discover that the Dirac-cone state of substrate $\mathrm{TIBiSe}_{2}$ migrates to the top surface of Pb film and obtains an energy gap below the superconducting transition temperature of $\mathrm{Pb}$. This suggests that a Bardeen-Cooper-Schrieffer superconductor is converted into a TSC by the topological proximity effect. Our discovery opens a route to manipulate topological superconducting properties of materials.

\footnotetext{
${ }^{1}$ Department of Physics, Tohoku University, Sendai 980-8578, Japan. ${ }^{2}$ Precursory Research for Embryonic Science and Technology (PRESTO), Japan Science and Technology Agency (JST), Tokyo 102-0076, Japan. ${ }^{3}$ Center for Spintronics Research Network, Tohoku University, Sendai 980-8577, Japan. ${ }^{4}$ WPI Research Center, Advanced Institute for Materials Research, Tohoku University, Sendai 980-8577, Japan. ${ }^{5}$ Institute of Scientific and Industrial Research, Osaka University, Ibaraki, Osaka 567-0047, Japan. ${ }^{6}$ Department of Physics, Kyoto Sangyo University, Kyoto 603-8555, Japan. ${ }^{7}$ Institute of Physics II, University of Cologne, Köln 50937, Germany. *email: t-sato@arpes.phys.tohoku.ac.jp
} 
T opological superconductors (TSCs) are a peculiar class of superconductors where the nontrivial topology of bulk leads to the emergence of Majorana bound states (MBSs) within the bulk superconducting gap ${ }^{1-5}$. Since MBSs are potentially applicable to the fault-tolerant quantum computation, searching for a new type of TSCs is one of the central challenges in quantum science. A straightforward way to realize TSCs would be to synthesize an odd-parity $p$-wave superconductor; however, intrinsic $p$-wave pairing is rare in nature, as highlighted by a limited number of $p$-wave superconductor candidates hitherto reported (e.g., refs. ${ }^{6,7}$ ). A different approach to realize TSCs is to utilize the superconducting proximity effect (SPE) in a heterostructure consisting of a conventional superconductor and a spinorbit coupled material such as a topological insulator (TI), as initiated by the theoretical prediction of effectively $p$-wave superconductivity induced in helical Dirac fermions and Rashba states ${ }^{8,9}$. This approach has been widely applied to various superconducting hybrids ${ }^{10-17}$, whereas the existence of MBSs is still under intensive debates. A part of the difficulty in establishing the SPE-derived topological superconductivity may lie in the SPE process itself, since the searched MBSs are expected to be localized in the vortex core at the interface within the heterostructure, and hence are hard to be accessed by surface-sensitive spectroscopies such as scanning tunneling microscopy (STM). Therefore, it would be desirable to invent an alternative way to realize TSCs without using bulk $p$-wave superconductor or the SPE.

In this work, we present the possibility to realize TSCs by using the topological proximity effect (TPE) $)^{18}$; such a novel approach was discovered through our angle-resolved photoemission (ARPES) study of a heterostructure consisting of an epitaxial $\mathrm{Pb}$ thin film grown on a three-dimensional (3D) TI, $\mathrm{TlBiSe}_{2}$.

\section{Results}

Fabrication and characterization of $\mathrm{Pb}$ film on $\mathrm{TlBiSe}_{2}$. The studies of SPE for generating TSCs have often employed a heterostructure consisting of a TI thin film as a top layer and a Bardeen-Cooper-Schrieffer (BCS) superconductor as a substrate $^{14-17}$. On the other hand, in our TPE approach, the stacking sequence is reversed, and a superconducting $\mathrm{Pb}$ thin film was grown on $\mathrm{TlBiSe}_{2}$ (Fig. 1a). We have deliberately chosen this combination, because (i) $\mathrm{Pb}$ films are known to maintain the superconductivity down to a few monolayers (MLs) ${ }^{19}$, and (ii) $\mathrm{TlBiSe}_{2}$ serves as a good substrate for epitaxial films ${ }^{18}$. Using the low-energy-electron-diffraction (LEED) (inset to Fig. 1d, f, h) and the ARPES results, we have estimated the in-plane lattice-constant $a$ to be 3.5 and $4.2 \AA$ for $\mathrm{Pb}(\sim 20 \mathrm{ML})$ and $\mathrm{TlBiSe}_{2}$, respectively. While the $a$ value of $\mathrm{Pb}$ film is close to that of bulk ${ }^{20}$, there is a sizable lattice mismatch of $19.5 \%$ between the $\mathrm{Pb}$ film and $\mathrm{TlBiSe}_{2}$.

First, we discuss the overall electronic structure. As shown in Fig. 1c, d, the electronic band structure of pristine $\mathrm{TlBiSe}_{2}$ is characterized by a Dirac-cone surface state (SS) around the $\bar{\Gamma}$ point that traverses the bulk valence and conduction bands ${ }^{21-23}$, forming a small Fermi surface (FS) centered at $\bar{\Gamma}$. Upon evaporation of $\mathrm{Pb}$ on $\mathrm{TlBiSe}_{2}$, the electronic structure drastically changes as seen in Fig. 1e, f; the holelike valence band of $\mathrm{TlBiSe}_{2}$ disappears, while several M-shaped bands emerge. The outermost holelike band crosses the Fermi level $\left(E_{\mathrm{F}}\right)$ and forms a large triangular FS (Fig. 1e). The M-shaped bands are ascribed to the quantum well states (QWSs) due to the quantum confinement of electrons in the $\mathrm{Pb}$ thin film. This is supported by the experimental fact that similar M-shaped bands are also observed in a $\mathrm{Pb}(111)$ thin film grown on $\mathrm{Si}(111)$ (Fig. 1h).
The QWSs in $\mathrm{Pb}$ thin films with various thickness on $\mathrm{Si}(111)$ have been well studied by spectroscopies and calculations ${ }^{19,24-28}$. Since the in-plane lattice constant of $\mathrm{Pb} / \mathrm{TlBiSe}_{2}$ is close to that of $\mathrm{Pb} / \mathrm{Si}(111)$, we expect a similar electronic structure between the two. By referring to the previous studies and our band-structure calculations, we estimated the film thickness to be $17 \mathrm{ML}$ for the case in Fig. 1e, f; see Supplementary Fig. 1 and Supplementary Note 1. We observed no obvious admixture from other MLs (e.g., 16 and $18 \mathrm{MLs}$ ) that would create additional QWSs ${ }^{24,26}$, suggesting an atomically flat nature of our $\mathrm{Pb}$ film. The LEED pattern of $17 \mathrm{ML}-\mathrm{Pb} / \mathrm{TlBiSe}_{2}$ as sharp as that of pristine $\mathrm{TlBiSe}_{2}$ (inset to Fig. 1f, d, respectively) also suggests the high crystallinity of $\mathrm{Pb}$ film. A careful look at Fig. If reveals an additional intensity spot near $E_{\mathrm{F}}$ above the topmost M-shaped band. This band is not attributed to the QWSs, and is responsible for our important finding, as described below.

Topological proximity effect. Next we clarify how the band structure of $\mathrm{TlBiSe}_{2}$ is influenced by interfacing with a $\mathrm{Pb}$ film. One may expect that there is no chance to observe the band structure associated with $\mathrm{TlBiSe}_{2}$ because the $\mathrm{Pb}$ film $(17 \mathrm{ML} \sim 5$ $\mathrm{nm})$ is much thicker than the photoelectron escape depth $(\sim 0.5-1 \mathrm{~nm})$. Figure 2a shows the ARPES-derived band structure near $E_{\mathrm{F}}$ obtained with a higher resolution for $17 \mathrm{ML}-\mathrm{Pb} / \mathrm{TlBiSe} \mathrm{C}_{2}$, where we clearly resolve an X-shaped band above the topmost QWSs. This band resembles the Dirac-cone SS in pristine $\mathrm{TlBiSe}_{2}$ (Fig. 2c), and is totally absent in $17 \mathrm{ML}-\mathrm{Pb} / \mathrm{Si}(111)$ (Fig. 2b), thereby ruling out the possibility of its $\mathrm{Pb}$ origin. The appearance of a Dirac-cone-like band in $17 \mathrm{ML}-\mathrm{Pb} / \mathrm{TlBiSe}_{2}$ is surprising, because the $\mathrm{Pb}$ film thickness is about ten times larger than the photoelectron escape depth. This in return definitely rules out the possibility that the observed Dirac-cone-like band is the Diraccone state embedded at the $\mathrm{Pb} / \mathrm{TlBiSe}_{2}$ interface. Furthermore, this band is not likely to originate from the accidentally exposed SS of TlBiSe $e_{2}$ through holes in $\mathrm{Pb}$, since the observed bands do not involve a replica of pristine $\mathrm{TlBiSe}_{2}$ bands and no trace of the $\mathrm{Tl}$ core-level peaks was found in $\mathrm{Pb} / \mathrm{TlBiSe}_{2}$; see Supplementary Fig. 2 and Supplementary Note 2. In fact, the bulk valence band lying below $0.4 \mathrm{eV}$ observed in pristine $\mathrm{TlBiSe}_{2}$ (Fig. 2c) totally disappears in $\mathrm{Pb} / \mathrm{TlBiSe}_{2}$, and in addition, the Dirac point of $\mathrm{Pb} /$ $\mathrm{TlBiSe}_{2}$ is shifted upward with respect to that of pristine $\mathrm{TlBiSe}_{2}$, as clearly seen in Fig. 2d-f. These observations led us to conclude that the Dirac-cone band has migrated from $\mathrm{TlBiSe}_{2}$ to the surface of $\mathrm{Pb}$ film via the TPE when interfacing $\mathrm{Pb}$ with $\mathrm{TlBiSe}_{2}{ }^{18}$. Such a migration can be intuitively understood in terms of the adiabatic bulk-band-gap reversal ${ }^{29,30}$ in the real space where the band gap (inverted gap) in $\mathrm{TlBiSe}_{2}$ closes throughout the gapless metallic overlayer and starts to open again at the $\mathrm{Pb}$-vacuum interface. It is noted that the upper branch of the Dirac-cone-like band would be connected to the quantized conduction band of the $\mathrm{Pb}$ film above $E_{\mathrm{F}}$ because it only crosses $E_{\mathrm{F}}$ once between $\Gamma$ and $\mathrm{M}$.

The band picture based on the TPE well explains the observed spectral feature in $\mathrm{Pb} / \mathrm{TlBiSe}_{2}$. As shown in Fig. $2 \mathrm{~g}-\mathrm{i}$, the spindegenerate topmost QWS of $\mathrm{Pb}$ (Fig. 2g) and the spin-polarized Dirac-cone SS of $\mathrm{TlBiSe}_{2}$ (Fig. 2h) start to interact each other when interfacing $\mathrm{Pb}$ and $\mathrm{TlBiSe}_{2}$. Due to the spin-selective band hybridization ${ }^{18}$, the Dirac-cone band is pushed upward, while the QWS is pulled down (Fig. 2i). This is exactly what we see in Fig. 2a. Our systematic thickness-dependent ARPES measurements revealed a detailed hybridization behavior between the Dirac-cone band and the QWSs, supporting this scenario; see Supplementary Fig. 3 and Supplementary Note 3. Noticeably, the migration of Dirac-cone state is observed at least up to $22 \mathrm{ML}$ thick ( $\sim .5 \mathrm{~nm}$ thick) $\mathrm{Pb}$ film. Such a long travel of the Dirac cone 
a

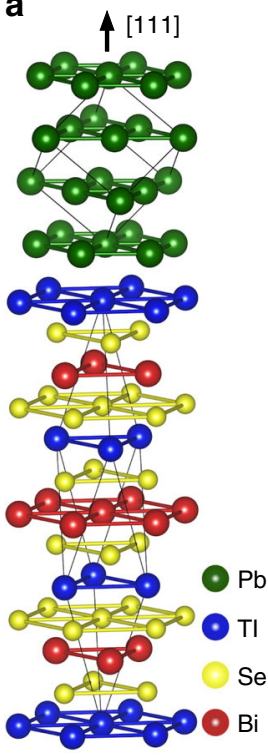

b

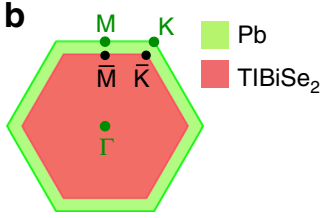

C
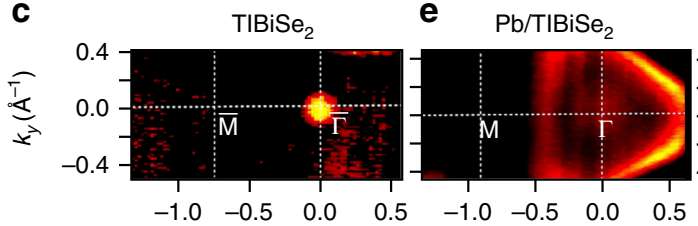

$k_{x}\left(\AA^{-1}\right)$

d

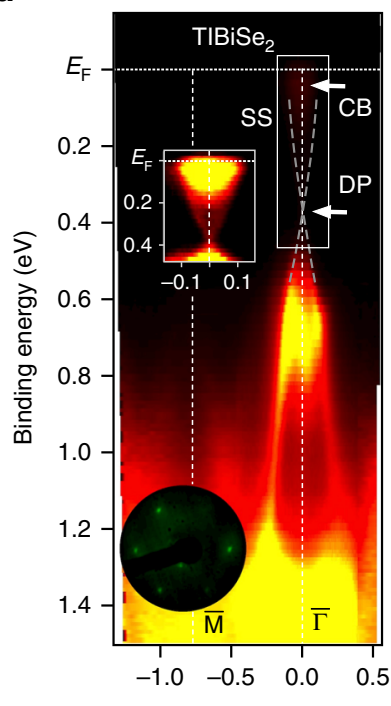

f

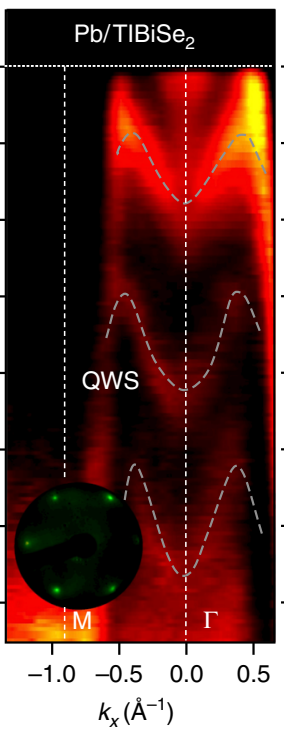

g $\mathrm{Pb} / \mathrm{Si}$

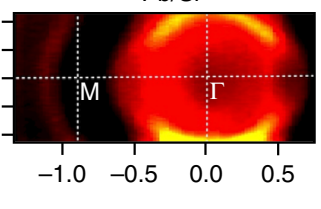

$\mathbf{h}$

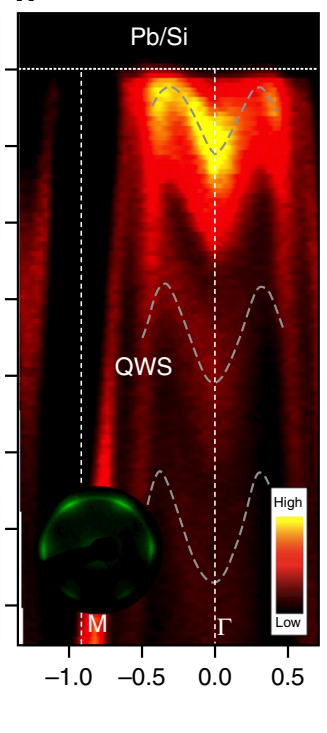

Fig. 1 Crystal and electronic structures of $\mathbf{P b}(\mathbf{1 1 1})$ thin film on TIBiSe $_{2}$. a Heterostructure consisting of $\mathrm{Pb}$ and TIBiSe $\mathrm{C}_{2}$. b Comparison of Brillouin zone between $\mathrm{TIBiSe}_{2}$ (red) and $\mathrm{Pb}$ (green). c Plot of ARPES intensity at $E_{\mathrm{F}}$ as a function of in-plane wave vector (namely, Fermi surface) around the $\overline{\Gamma M}$ line for pristine $\mathrm{TIBiSe}_{2}$, measured with the He-l $\alpha$ line $(h \nu=21.218 \mathrm{eV})$. d ARPES-derived band structure along the $\overline{\Gamma M}$ cut for pristine TIBiSe 2 . Inset shows the ARPES intensity with enhanced color contrast in the area enclosed by white rectangle. e, $\mathbf{f}$ Same as $\mathbf{c}$ and $\mathbf{d}$ but for $17 \mathrm{ML}-\mathrm{Pb} / \mathrm{TIBiSe} \mathrm{e}_{2} . \mathbf{g}$, $\mathbf{h} \mathrm{Same}$ as $\mathbf{e}$ and f but for $17 \mathrm{ML}-\mathrm{Pb} / \mathrm{Si}(111)$. SS, CB, DP, and QWS denote the surface state, conduction band, Dirac point, and quantum well state, respectively. Arrows in $\mathbf{d}$ indicate the location of CB and DP. Dashed gray curves are a guide for the eyes to trace the SS and QWSs. Insets to $\mathbf{d}, \mathbf{f}$, and $\mathbf{h}$ are the LEED patterns of the respective films.

in the real space is unexpected, and hard to be reproduced by the band calculations due to large incommensurate lattice mismatch between $\mathrm{Pb}$ and $\mathrm{TlBiSe}_{2}$. In fact, we have tried to calculate the band dispersion of $\mathrm{Pb} / \mathrm{TlBiSe}_{2}$ slab by expanding the in-plane lattice constant of $\mathrm{Pb}$ film to hypothetically form a commensurate system, but it caused a sizable change in the whole band structure of $\mathrm{Pb}$ film, resulting in the band structure totally different from the experiment. Alternatively, a calculation that uses a larger inplane unit cell might be useful to achieve an approximate lattice match between $\mathrm{Pb}$ and $\mathrm{TlBiSe}_{2}$. It is noted here that the coherency of electronic states may play an important role for the observation of a coupling with the substrate (i.e., the TPE in this study) as in the case of other quantum composite systems involving metallic overlayer ${ }^{31}$. We estimate the electronic coherence length in $\mathrm{Pb}$ film to be larger than $22 \mathrm{ML}(\sim 6.5 \mathrm{~nm})$ because the topological SS is observed even in the $22 \mathrm{ML}$ film; see Supplementary Fig. 3 and Supplementary Note 3.

Superconducting gap. The next important issue is whether the $\mathrm{Pb} / \mathrm{TlBiSe}_{2}$ heterostructure hosts superconductivity. To elucidate it, we first fabricated a thicker (22 ML) Pb film on $\mathrm{TlBiSe}_{2}$ and carried out ultrahigh-resolution ARPES measurements at low temperatures. Figure $3 \mathrm{~b}$ shows the energy distribution curve (EDC) at the $\mathbf{k}_{\mathrm{F}}$ point of the $\mathrm{Pb}$-derived triangular FS (point $\mathrm{A}$ in Fig. 3a) measured at $T=4$ and $10 \mathrm{~K}$ across the superconducting transition temperature $T_{\mathrm{c}}$ of bulk $\mathrm{Pb}(7.2 \mathrm{~K})$. At $T=4 \mathrm{~K}$, one can clearly recognize a leading-edge shift toward higher $E_{\mathrm{B}}$ together with a pile up in the spectral weight, a typical signature of the superconducting-gap opening. This coherence peak vanishes at $T=10 \mathrm{~K}$ due to the gap closure, as better visualized in the symmetrized EDC (Fig. 3c). We have estimated the superconducting-gap size at $T=4 \mathrm{~K}$ to be $1.3 \mathrm{meV}$ from the numerical fittings. This value is close to that of bulk $\mathrm{Pb}(\sim 1.2$ $\mathrm{meV})^{32}$, suggesting that the $T_{\mathrm{c}}$ is comparable to that of bulk $\mathrm{Pb}$.

Since the superconductivity shows up on the $\mathrm{Pb}$ film, we now address an essential question whether the migrating Dirac-cone band hosts superconductivity. We show in Fig. 3d-i the EDCs and corresponding symmetrized EDCs at $T=4$ and $10 \mathrm{~K}$ for the $17 \mathrm{ML}$ sample measured at three representative $\mathbf{k}_{\mathrm{F}}$ points (points $\mathrm{A}-\mathrm{C}$ in Fig. 3a). At point $\mathrm{A}$ on the $\mathrm{Pb}$-derived $\mathrm{FS}$, we observe the superconducting-gap opening (Fig. 3d), similarly to the $22 \mathrm{ML}$ film. At point B (C), where the migrating Dirac-cone band crosses $E_{\mathrm{F}}$ along the $\overline{\Gamma K}(\overline{\Gamma M})$ line, we still observe a gap as seen in Fig. $3 \mathrm{f}$ (Fig. 3h). This indicates that an isotropic superconducting gap opens on the migrating Dirac-cone FS. We observed that this gap persists at least down to $12 \mathrm{ML}$, confirming that the superconducting gap is not an artifact that accidentally appears at some specific film thickness; see Supplementary Fig. 4 and Supplementary Note 4 . We have also confirmed that the gap opening is not an inherent nature of the original topological SS in pristine $\mathrm{TlBiSe}_{2}$, by observing no leading-edge shift or spectral-weight suppression at $E_{\mathrm{F}}$ at $4 \mathrm{~K}$ in pristine $\mathrm{TlBiSe}_{2}$ (Fig. 3j, k).

\section{Discussion}

The present results show that the superconducting gap opens on the entire FS originating from both the Pb-derived QWSs and the 


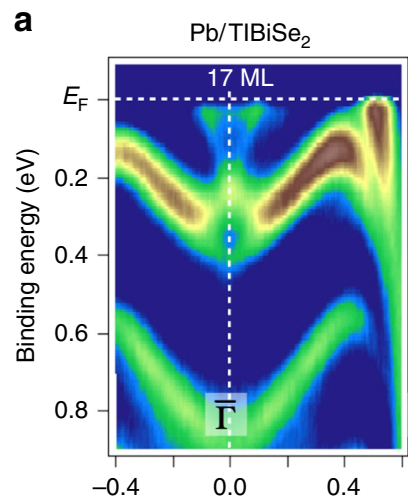

b

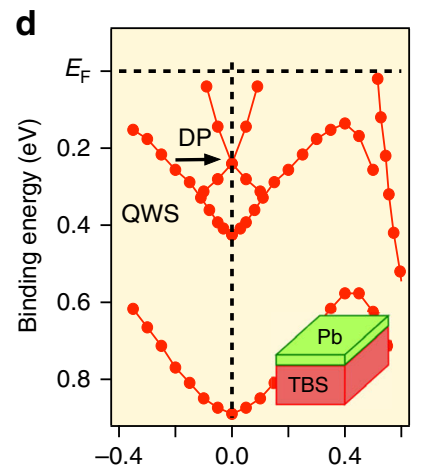

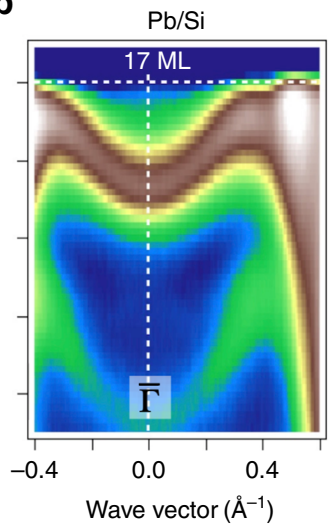

Wave vector $\left(\AA^{-1}\right)$

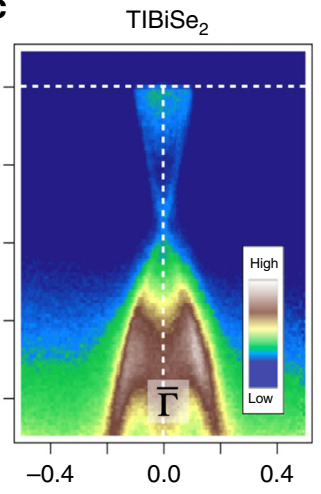

e
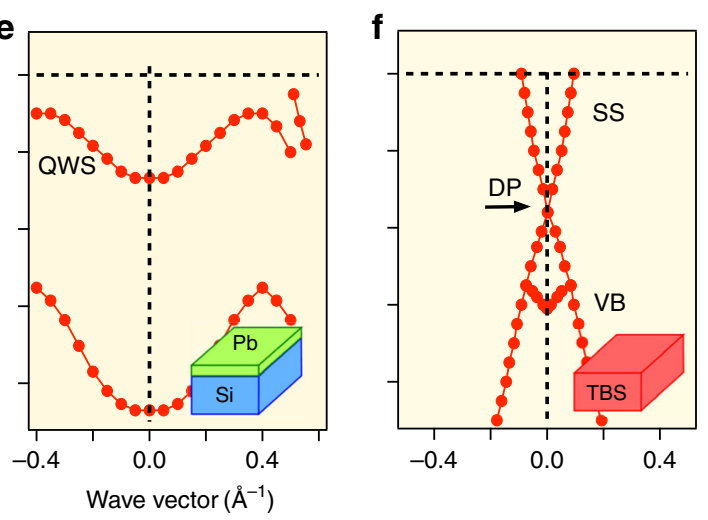

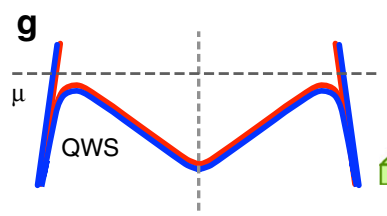

$\bar{\Gamma}$

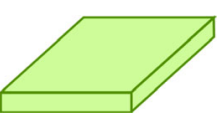

$17 \mathrm{ML} \mathrm{Pb}$
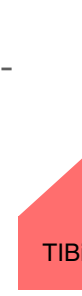
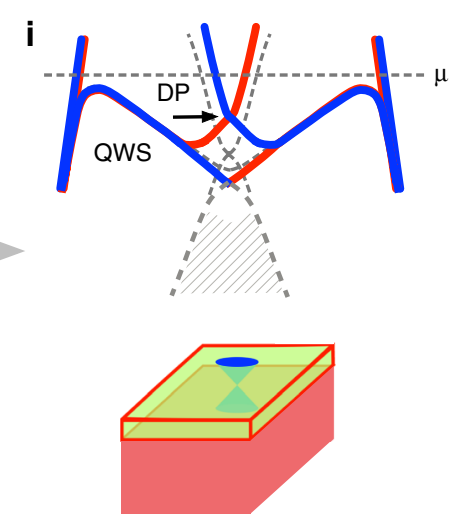

Fig. 2 Migration of topological surface states to the surface of $\mathbf{P b}$ film. a-c ARPES-derived band structure near $E_{\mathrm{F}}$ around the $\bar{\Gamma}$ point for $17 \mathrm{ML}-\mathrm{Pb} /$ $\mathrm{TIBiSe}_{2}, 17 \mathrm{ML}-\mathrm{Pb} / \mathrm{Si}(111)$, and pristine $\mathrm{TIBiSe}{ }_{2}$, respectively, with the $\mathrm{He}-\mathrm{l} \alpha$ line. The data were obtained along the $\overline{\Gamma K}$ cut parallel to the analyzer slit, so that the energy and momentum resolutions are better than the data in Fig. 1d, f, h measured along the $\bar{\Gamma} \bar{M}$ cut perpendicular to the analyzer slit. d-f Experimental band dispersions extracted from the peak positions of MDCs/EDCs for a-c. g-i Schematics of the hybridization between topological Diraccone state and QWSs, showing the migration of topological Dirac-cone state upon interfacing Pb with $\mathrm{TlBiSe}_{2}$. Dashed curves in $\mathbf{i}$ indicate the band dispersion without hybridization. Note that the bulk $\mathrm{VB}$ of $\mathrm{TIBiSe}_{2}$ indicated by gray shade becomes invisible on the $\mathrm{Pb} / \mathrm{TIBiSe} \mathrm{C}_{2}$ interface, because only the topological SS migrates to the top surface.

migrating Dirac-cone band (Fig. 31). The emergence of an isotropic superconducting gap on the Dirac-cone FS suggests that the $2 \mathrm{D}$ topological superconductivity is likely to be realized, since this heterostructure satisfies the theoretically proposed condition for the effectively $p$-wave superconducting helical-fermion state ${ }^{8}$. In this regard, one may think that such realization is a natural consequence of making heterojunction between superconductor and TI. However, the present study proposes an essentially new strategy to realize the $2 \mathrm{D}$ topological superconductivity. In the ordinary approach based on the SPE (Fig. $3 \mathrm{~m}$ ), the topological Dirac-cone state hosts the effective $p$-wave pairing at the interface due to the penetration of Cooper pairs from the superconductor to the TI. On the other hand, the present approach does not rely on this phenomenon at all, because the topological Dirac-cone state appears on the top surface of a superconductor (Fig. 3n) via the TPE.

One can view this effect as a conversion of a conventional superconductor ( $\mathrm{Pb}$ film without topological $\mathrm{SS})$ to a TSC $(\mathrm{Pb}$ film with topological SS) by interfacing. The present approach to realize $2 \mathrm{D}$ TSCs has an advantage in the sense that the pairing in the helical-fermion state (and the MBS as well) is directly accessed by surface spectroscopies such as STM and ARPES. The superconducting helical fermions would be otherwise embedded deep at the interface and are hard to be 


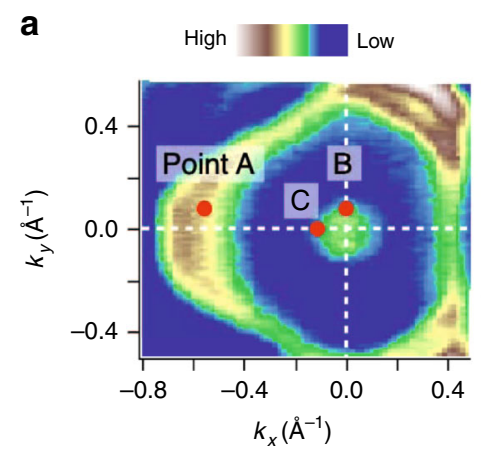

I
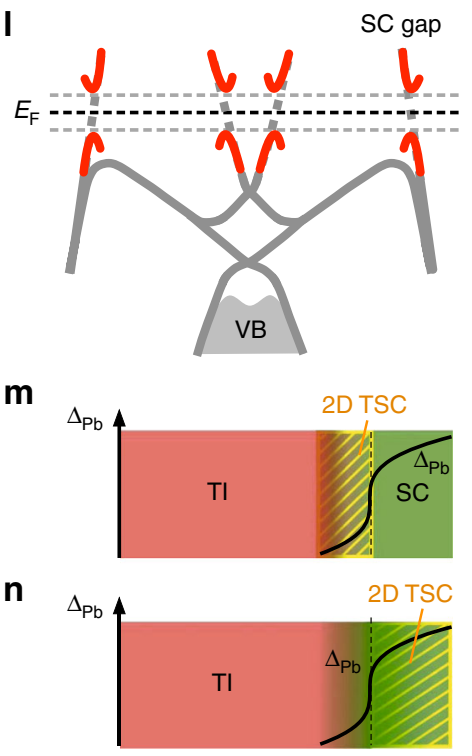

b
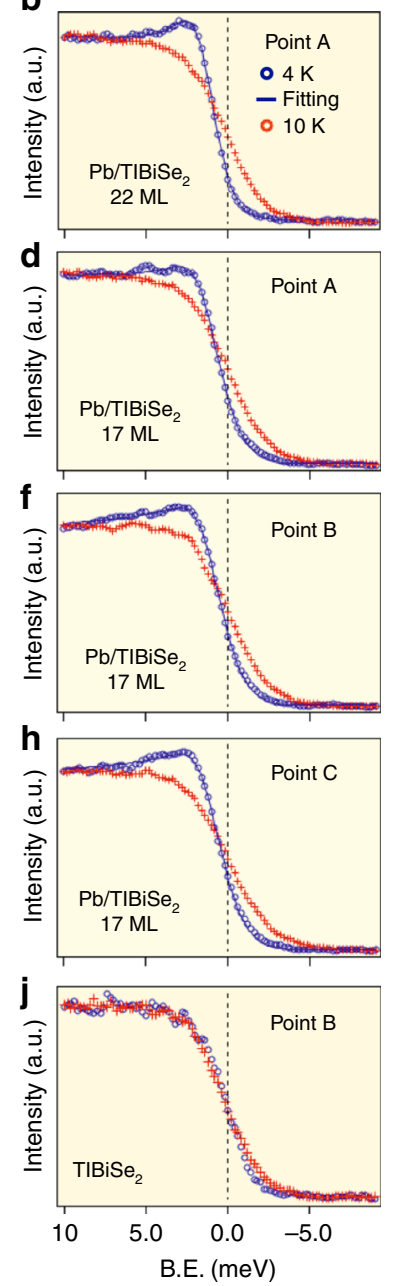

c
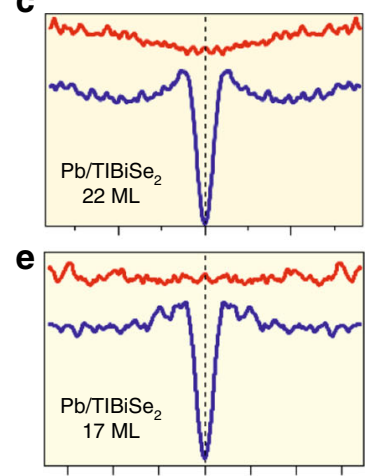

g

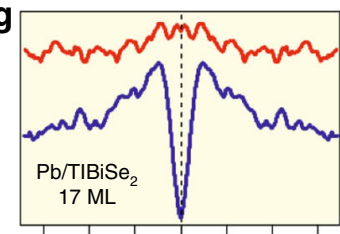

i
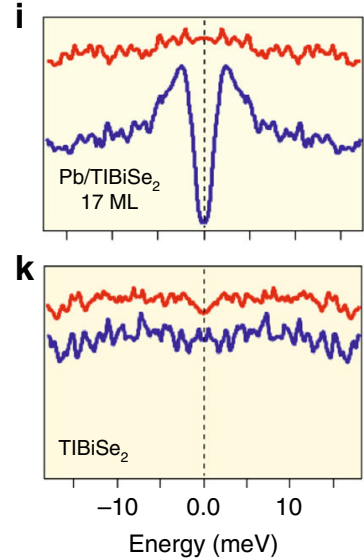

Fig. 3 Possible topological superconductivity in $\mathbf{P b}$ film. a ARPES-intensity mapping at $E_{\mathrm{F}}$ for $22 \mathrm{ML}-\mathrm{Pb} / \mathrm{TIBiSe} \mathrm{e}_{2}$. b, $\mathbf{c}$ Ultrahigh-resolution EDCs and corresponding symmetrized EDCs, respectively, at $T=4$ and $10 \mathrm{~K}$, measured at point $\mathrm{A}$ in $\mathbf{a}$ ( $\mathbf{k}_{\mathrm{F}}$ point of the QWS) for $22 \mathrm{ML}-\mathrm{Pb} / \mathrm{TIBiSe}$. $\mathbf{d}, \mathbf{e} \mathrm{Same}$ as $\mathbf{b}$ and $\mathbf{c}$, respectively, but for $17 \mathrm{ML}-\mathrm{Pb} / \mathrm{TIBiSe}_{2} . \mathbf{f}, \mathbf{g}$ Same as $\mathbf{d}$ and $\mathbf{e}$, respectively, but measured at point B ( $\mathbf{k}_{\mathrm{F}}$ point of the topological SS). $\mathbf{h}, \mathbf{i}$ Same as $\mathbf{d}$ and $\mathbf{e}$, respectively, but measured at point $C$. j, $\mathbf{k}$ Same as $\mathbf{f}$ and $\mathbf{g}$, respectively, but for pristine $\mathrm{TIBiSe}_{2}$. Blue solid curve in the EDC at $T=4 \mathrm{~K}$ in $\mathbf{b}, \mathbf{d}, \mathbf{f}$, and $\mathbf{h}$ is the result of numerical fittings using the Dynes function multiplied by the Fermi-Dirac distribution function, convoluted with a resolution function. I Illustration of the superconducting-gap opening on the QWS- and TSS-derived bands. $\mathbf{m}$ Conventional view on the realization of 2D TSC via SPE. $\mathbf{n}$ Proposed method to realize 2D TSC by converting a conventional superconductor into a TSC through the TPE.

accessed if the TPE does not occur. Moreover, the observed gap magnitude on the topological SS is comparable to that of the original $\mathrm{Pb}$, unlike the SPE-induced gap that is usually smaller. This result tells us that the so-far overlooked TPE had better be seriously taken into account in many superconductor-TI hybrids. Also, the present study points to the possibility of realizing even wider varieties of $2 \mathrm{D}$ TSCs by using the TPE. It is noted that the topological states in $\mathrm{Pb} / \mathrm{TlBiSe}_{2}$ are electrically shorted out by the metallic QWSs, unlike the case of some TI films on top of superconductors. This needs to be considered in the application because single conducting channel from the Dirac-cone states would be more preferable. In this regard, the present approach utilizing the TPE and the existing approach using the SPE would be complementary to each other.

\section{Methods}

Sample preparation. High-quality single crystals of $\mathrm{TlBiSe}_{2}$ were grown by a modified Bridgman method ${ }^{21}$. To prepare a $\mathrm{Pb}$ film, we first cleaved a $\mathrm{TlBiSe}_{2}$ crystal under ultrahigh vacuum with scotch tape to obtain a shiny mirror-like surface, and then deposited $\mathrm{Pb}$ atoms (purity; $5 \mathrm{~N}$ ) on the $\mathrm{TlBiSe}_{2}$ substrate using the molecular-beam epitaxy technique while keeping the substrate temperature at $T=85 \mathrm{~K} . \mathrm{A} \mathrm{Pb}(111)$ film on $\mathrm{Si}(111)$, used as a reference, was fabricated by keeping the same substrate temperature. The film thickness was controlled by the deposition time at a constant deposition rate. The actual thickness was estimated by a comparison of ARPES-derived band dispersions with the band-structure calculations for free-standing multilayer $\mathrm{Pb}$.

ARPES measurements. ARPES measurements were performed with the MBS-A1 electron analyzer equipped with a high-intensity He discharge lamp. After the growth of $\mathrm{Pb}$ thin film by evaporation, it was immediately transferred to the sample cryostat kept at $T=30 \mathrm{~K}$ in the ARPES chamber, to avoid the clusterization of $\mathrm{Pb}$ that is accelerated at room temperature (note that such clusterization hinders the detailed investigation of the surface morphology by atomic-force microscopy). We used the He-Ia resonance line $(h v=21.218 \mathrm{eV})$ to excite photoelectrons. The energy resolution of ARPES measurements was set to be $2-40 \mathrm{meV}$. The sample temperature was kept at $T=30 \mathrm{~K}$ during the ARPES-intensity-mapping measurements, while $T=4$ and $10 \mathrm{~K}$ for the superconducting-gap measurements. The Fermi level $\left(E_{\mathrm{F}}\right)$ of the samples was referenced to that of a gold film evaporated onto the sample holder.

Band calculations. First-principles band-structure calculations were carried out by a projector augmented wave method implemented in Vienna Ab initio Simulation Package code ${ }^{33}$ with generalized gradient approximation potential ${ }^{34}$. After the crystal structure was fully optimized, the spin-orbit coupling was included selfconsistently. 


\section{Data availability}

The data sets generated/analyzed during the current study are available from the corresponding author on reasonable request.

Received: 24 July 2019; Accepted: 10 December 2019;

Published online: 09 January 2020

\section{References}

1. Hasan, M. Z. \& Kane, C. L. Colloquium: topological insulators. Rev. Mod. Phys. 82, 3045-3067 (2010).

2. Qi, X. L. \& Zhang, S. C. Topological insulators and superconductors. Rev. Mod. Phys. 83, 1057-1110 (2011).

3. Ando, Y. \& Sato, M. Topological superconductors: a review. Rep. Prog. Phys. 80, 076501 (2017).

4. Alicea, J. New directions in the pursuit of Majorana fermions in solid state systems. Rep. Prog. Phys. 75, 076501 (2012).

5. Beenakker, C. W. J. Search for Majorana Fermions in superconductors. Annu. Rev. Condens. Matter Phys. 4, 113 (2013).

6. Maeno, Y. et al. Superconductivity in a layered perovskite without copper. Nature 372, 532-534 (1994).

7. Hor, Y. S. et al. Superconductivity in $\mathrm{Cu}_{x} \mathrm{Bi}_{2} \mathrm{Se}_{3}$ and its implications for pairing in the undoped topological insulator. Phys. Rev. Lett. 104, 057001 (2010).

8. Fu, L. \& Kane, C. L. Superconducting proximity effect and Majorana fermions at the surface of a topological insulator. Phys. Rev. Lett. 100, 096407 (2008).

9. Sato, M. \& Fujimoto, S. Topological phases of noncentrosymmetric superconductors: edge states, Majorana fermions, and non-Abelian statistics. Phys. Rev. B 79, 094504 (2009).

10. Mourik, V. et al. Signatures of Majorana fermions in hybrid superconductorsemiconductor nanowire devices. Science 336, 1003-1007 (2012).

11. Das, A. et al. Zero-bias peaks and splitting in an Al-InAs nanowire topological superconductor as a signature of Majorana fermions. Nat. Phys. 8, 887-895 (2012).

12. Deng, M. T. et al. Majorana bound state in a coupled quantum-dot hybridnanowire system. Science 354, 1557-1562 (2016).

13. Nadj-Perge, S. et al. Observation of Majorana fermions in ferromagnetic atomic chains on a superconductor. Science 346, 602-607 (2014).

14. Wang, M. et al. The coexistence of superconductivity and topological order in the $\mathrm{Bi}_{2} \mathrm{Se}_{3}$ thin films. Science 336, 52-55 (2012).

15. Xu, S.-Y. et al. Momentum-space imaging of Cooper pairing in a half-Diracgas topological superconductor. Nat. Phys. 10, 943-950 (2014).

16. Sun, H.-H. et al. Coexistence of topological edge state and superconductivity in bismuth ultrathin film. Nano Lett. 17, 3035-3039 (2017).

17. Flötotto, D. et al. Superconducting pairing of topological surface states in bismuth selenide films on niobium. Sci. Adv. 4, eaar7214 (2018).

18. Shoman, T. et al. Topological proximity effect in a topological insulator hybrid. Nat. Commun. 6, 6547 (2015).

19. Qin, S., Kim, J. \& Shih, C.-K. Superconductivity at the two-dimensional limit. Science 324, 1314-1317 (2009).

20. Davey, W. P. Precision measurements of the lattice constants of twelve common metals. Phys. Rev. 25, 753 (1925).

21. Sato, T. et al. Direct evidence for the Dirac-cone topological surface states in the ternary chalcogenide $\mathrm{TlBiSe}_{2}$. Phys. Rev. Lett. 105, 136802 (2010).

22. Kuroda, K. et al. Experimental realization of a three-dimensional topological insulator phase in ternary chalcogenide TlBiSe 2 . Phys. Rev. Lett. 105, 146801 (2010).

23. Chen, Y. L. et al. Single Dirac cone topological surface state and unusual thermoelectric property of compounds from a new topological insulator family. Phys. Rev. Lett. 105, 266401 (2010).

24. Wei, C. M. \& Chou, M. Y. Theory of quantum size effects in thin $\mathrm{Pb}(111)$ films. Phys. Rev. B 66, 233408 (2002).

25. Man, A., Dil, J. H., Ettema, A. R. H. F. \& Weitering, H. H. Quantum electronic stability and spectroscopy of ultrathin $\mathrm{Pb}$ films on $\mathrm{Si}(111) 7 \times 7$. Phys. Rev. B 66 , 195410 (2002).

26. Upton, M. H., Miller, T. \& Chiang, T.-C. Absolute determination of film thickness from photoemission: application to atomically uniform films of $\mathrm{Pb}$ on Si. Appl. Phys. Lett. 85, 1235-1237 (2004).

27. Upton, M. H., Wei, C. W., Chou, M. Y., Miller, T. \& Chiang, T.-C. Thermal stability and electronic structure of atomically uniform $\mathrm{Pb}$ films on $\mathrm{Si}(111)$. Phys. Rev. Lett. 93, 026802 (2004).
28. Zhang, Y.-F. et al. Band structure and oscillatory electron-phonon coupling of $\mathrm{Pb}$ thin films determined by atomic-layer-resolved quantum-well states. Phys. Rev. Lett. 95, 096802 (2005).

29. Wang, X., Bian, G., Miller, T. \& Chiang, T.-C. Topological spin-polarized electron layer above the surface of Ca-terminated $\mathrm{Bi}_{2} \mathrm{Se}_{3}$. Phys. Rev. B 87, 035109 (2013).

30. Wang, X., Bian, G., Miller, T. \& Chiang, T.-C. Topological quantum well resonances in metal overlayers. Phys. Rev. B 87, 235113 (2013).

31. Liu, Y. et al. Interface-induced complex electronic interference structures in Ag films on Ge(111). Phys. Rev. B 78, 035443 (2008).

32. Chainani, A., Yokoya, T., Kiss, T. \& Shin, S. Photoemission spectroscopy of the strong-coupling superconducting transitions in lead and niobium. Phys. Rev. Lett. 85, 1966-1969 (2000)

33. Kresse, G. \& Furthmüller, J. Efficient iterative schemes for ab-initio total energy calculations using a plane-wave basis set. Phys. Rev. B 54, 11169-11186 (1996).

34. Perdew, J. P., Burke, K. \& Ernzerhof, M. Generalized gradient approximation made simple. Phys. Rev. Lett. 77, 3865-3868 (1996).

\section{Acknowledgements}

We thank T.N., K.H., A.T. and T.R. for their assistance in the ARPES experiments. This work was supported by Grant-in-Aid for Scientific Research on Innovative Areas "Topological Materials Science" (JSPS KAKENHI Grant numbers JP15H05853, JP18H04227, and JP15K21717), JST-CREST (no. JPMJCR18T1), JST-PRESTO (no. JPMJPR18L7), and Grant-in-Aid for Scientific Research (JSPS KAKENHI Grant numbers JP17H01139, JP15H02105, JP26287071, and JP25287079). The work in Cologne was funded by the Deutsche Forschungsgemeinschaft (German Research Foundation) Project number 277146847 - CRC 1238 (Subproject A04).

\section{Author contributions}

The work was planned and proceeded by discussion among C.X.T., K.N., S.S., K.S., T.S. T.T., and Y.A. K.S. carried out the growth of bulk single crystals and their characterization. C.X.T and I.W. fabricated ultrathin films. C.X.T., N.S., and I.W. performed the ARPES measurements. K.Y. and T.O. carried out the band calculations. C.X.T. and T.S finalized the manuscript with inputs from all the authors.

\section{Competing interests}

The authors declare no competing interests.

\section{Additional information}

Supplementary information is available for this paper at https://doi.org/10.1038/s41467 019-13946-0.

Correspondence and requests for materials should be addressed to T.S

Peer review information Nature Communications thanks Tian Qian and the other, anonymous, reviewer(s) for their contribution to the peer review of this work. Peer reviewer reports are available.

Reprints and permission information is available at http://www.nature.com/reprints

Publisher's note Springer Nature remains neutral with regard to jurisdictional claims in published maps and institutional affiliations.

Open Access This article is licensed under a Creative Commons Attribution 4.0 International License, which permits use, sharing, adaptation, distribution and reproduction in any medium or format, as long as you give appropriate credit to the original author(s) and the source, provide a link to the Creative Commons license, and indicate if changes were made. The images or other third party material in this article are included in the article's Creative Commons license, unless indicated otherwise in a credit line to the material. If material is not included in the article's Creative Commons license and your intended use is not permitted by statutory regulation or exceeds the permitted use, you will need to obtain permission directly from the copyright holder. To view a copy of this license, visit http://creativecommons.org/ licenses/by/4.0/

(C) The Author(s) 2020 